\title{
A importância do fortalecimento da musculatura do assoalho pélvico na saúde da
}

\section{mulher}

\author{
The importance of strengthening the pelvic floor muscles in women's health \\ La importancia de fortalecer la musculatura del suelo pélvico en la salud de la mujer
}

Recebido: 12/02/2021 | Revisado: 18/02/2021 | Aceito: 21/02/2021 | Publicado: 28/02/2021

\author{
Bruna Pereira Nagamine \\ ORCID: https://orcid.org/0000-0002-8799-2529 \\ Instituto Educacional Santa Catarina, Brasil \\ E-mail: brunapnagamine@gmail.com \\ Rildo da Silva Dantas \\ ORCID: https://orcid.org/0000-0001-5684-979X \\ Instituto Educacional Santa Catarina, Brasil \\ E-mail: rildodantas93@gmail.com \\ Karla Camila Correia da Silva \\ ORCID: https://orcid.org/0000-0003-1538-7028 \\ Instituto Educacional Santa Catarina, Brasil \\ Faculdade Guaraí, Brasil \\ E-mail: karlacamilac@yahoo.com.br
}

\begin{abstract}
Resumo
A fisioterapia na saúde da mulher vem ganhando destaque na prevenção e no tratamento da diminuição da força do assoalho pélvico, que com o enfraquecimento da musculatura do assolho pélvico a mulher pode desenvolver disfunções sexuais, incontinências, prolapso genital e flacidez vaginal. O objetivo do presente artigo é demostrar a importância do fortalecimento da musculatura do assolho pélvico para prevenir disfunções causadas pelo enfraquecimento da musculatura do assoalho. O estudo é uma revisão de literatura de caráter descritivo e quantitativo, pesquisado em bases de dados como Cochrane Library, Scientific Eletronic Library Online (SciELO) e livros com descritores, "Disfunções sexuais", "Assoalho Pélvico", "Gravidez". No decorrer da vida as mulheres passam por fases e períodos específicos que são determinados por mudanças físicas, hormonais e psicológicas, e o enfraquecimento da musculatura do assoalho pélvico é consequência dessas mudanças, podendo levar a mulher a desenvolver: disfunções sexuais, incontinências tanto urinaria quanto fecal, prolapso genital, insatisfação sexual e alterações nas respostas sexuais. Percebe-se que o fortalecimento da musculatura do assoalho pélvico, atua tanto na prevenção quanto no tratamento dessas disfunções e tem como objetivo melhorar a força muscular do assoalho prevenindo disfunções e alterações e através do fortalecimento dessa musculatura, se tem a melhora da força muscular, ganho de mobilidade pélvica e aumento da sensibilidade clitoriana e perineal e aumento do fluxo sanguíneo melhorando as fases da resposta sexual, da lubrificação, da excitação, do desejo e do orgasmo, reduzindo as tensões musculares, favorecendo o relaxamento da musculatura, gerando consciência corporal e qualidade sexual.
\end{abstract}

Palavras-chave: MAP; Fortalecimento muscular; Saúde da mulher.

\begin{abstract}
Physiotherapy in women's health has been gaining prominence in the prevention and treatment of decreased strength of the pelvic floor, which, with the weakening of the pelvic muscle, the woman can develop sexual dysfunctions, incontinence, genital prolapse and vaginal flaccidity. The purpose of this article is to demonstrate the importance of strengthening the pelvic ridge muscles to prevent dysfunctions caused by the weakening of the floor muscles. The study is a literature review of a descriptive and quantitative character, searched in databases such as Cochrane Library, Scientific Electronic Library Online (SciELO) and books with descriptors, "Sexual dysfunctions", "Pelvic Floor", "Pregnancy". Throughout their lives, women go through specific phases and periods that are determined by physical, hormonal and psychological changes, and the weakening of the pelvic floor muscles is a consequence of these changes, which may lead the woman to develop: sexual dysfunction, urinary incontinence as well as fecal, genital prolapse, sexual dissatisfaction and changes in sexual responses. It is perceived that the strengthening of the pelvic floor musculature, acts both in the prevention and in the treatment of these dysfunctions and aims to improve the muscular strength of the floor preventing dysfunctions and alterations and through the strengthening of this musculature, if there is an improvement in muscle strength, gain of pelvic mobility and increased clitoral and perineal sensitivity and increased blood flow improving the phases of sexual response, lubrication, arousal, desire and orgasm, reducing muscle tension, favoring muscle relaxation, generating body awareness and sexual quality.
\end{abstract}

Keywords: MAP; Muscle strengthening; Women's health. 


\section{Resumen}

La fisioterapia en la salud de la mujer ha ido ganando protagonismo en la prevención y tratamiento de la disminución de la fuerza del suelo pélvico, que, con el debilitamiento del músculo pélvico, la mujer puede desarrollar disfunciones sexuales, incontinencia, prolapso genital y flacidez vaginal. El propósito de este artículo es demostrar la importancia de fortalecer los músculos de la cresta pélvica para prevenir disfunciones causadas por el debilitamiento de los músculos del piso. El estudio es una revisión de la literatura de carácter descriptivo y cuantitativo, buscada en bases de datos como Cochrane Library, Scientific Electronic Library Online (SciELO) y libros con descriptores, "Disfunciones sexuales", "Suelo pélvico", "Embarazo". A lo largo de su vida, la mujer pasa por fases y periodos específicos que vienen determinados por cambios físicos, hormonales y psicológicos, y el debilitamiento de la musculatura del suelo pélvico es consecuencia de estos cambios, que pueden llevar a la mujer a desarrollar: disfunción sexual, incontinencia urinaria así como prolapso fecal, genital, insatisfacción sexual y cambios en las respuestas sexuales. Se percibe que el fortalecimiento de la musculatura del piso pélvico, actúa tanto en la prevención como en el tratamiento de estas disfunciones y tiene como objetivo mejorar la fuerza muscular del piso previniendo disfunciones y alteraciones y mediante el fortalecimiento de esta musculatura, si existe una mejora de la fuerza muscular, ganancia de movilidad pélvica y aumento de la sensibilidad del clítoris y perineal y aumento del flujo sanguíneo mejorando las fases de respuesta sexual, lubricación, excitación, deseo y orgasmo, reduciendo la tensión muscular, favoreciendo la relajación muscular, generando conciencia corporal y calidad sexual.

Palabras clave: MAP; Fortalecimiento muscular; La salud de la mujer.

\section{Introdução}

Cada vez mais a saúde da mulher vem sendo como parte da saúde global e bem-estar da mulher, que no decorrer da vida as mulheres passam por fases e períodos específicos que são determinados por mudanças físicas, hormonais e psicológicas. Como por exemplo a descoberta e o desenvolvimento sexual do corpo e a gravidez que é um processo fisiológico onde ocorrem muitas alterações principalmente hormonais que acarretam mudanças físicas, psíquicas e comportamentais na mulher. Durante a gestação, há uma sobrecarga da musculatura do assoalho pélvico (MAP), pois, promove a sustentação dos órgãos pélvicos e dos anexos embrionários e o peso do bebê, e com o progresso da gravidez o assoalho pélvico (AP) sofre uma sobrecarga fazendo diminuir a força muscular, devido às alterações biomecânicas e hormonais normais da gestação, devido a isso e outros fatores como: alteração postural, ganho excessivo de peso, hábitos urinários inadequados podem levar a gestante a desenvolver alguma disfunção. (Polden \& Mantle, 2002; Souza, 2002).

$\mathrm{Na}$ parte interna da pelve se localiza a musculatura do assoalho pélvico que é formado por fáscias, ligamentos e músculos, e tem como objetivo promover a sustentação dos órgãos internos, principalmente a bexiga, o útero e o reto (Baracho, 2014).

O assoalho pélvico é composto pelos músculos levantadores do ânus (pubovisceral puboccígeo, e ileococcígeo), coccígeo, bulbocavernoso, isquiocavernoso, e músculo perineal transverso profundo e superficial. O MAP pode ser exercitada em todas as fases vida da mulher mais em principal na gestação, considerando-se que o períneo enfraquecido pode causar disfunções sexuais. A musculatura perineal enfraquecida pode levar a incontinência urinária, prolapso genital, disfunção sexual e flacidez vaginal (Baracho, 2007).

A atuação do fisioterapeuta no fortalecimento e na reeducação perineal do assoalho pélvico, tem como objetivo melhorar a força de contração das fibras musculares, prevenir disfunções sexuais e incontinências. (Nolasco et al., 2008). Com isso, os exercícios de fortalecimento para o assoalho pélvico são eficazes para melhorar a força da musculatura, a sensibilidade, aumentar o fluxo sanguíneo da região perineal, favorecendo a melhora do desejo sexual, orgasmo, da lubrificação, e da excitação diante disso fortalecer o assoalho pélvico previne incontinência urinaria, disfunções sexuais, prolapso genital e melhora a satisfação e a qualidade sexual (Etienne; Waitman, 2006). 


\section{Metodologia}

Este estudo trata-se de uma revisão bibliográfica, descritiva e exploratória, de abordagem qualitativa. Tendo como base para sua discussão teórica, artigos científicos e demais produções intelectuais (Pereira et al., 2018).

Foram considerados os critérios de inclusão: artigos e produções intelectuais publicadas preferencialmente nos últimos 10 anos, compreendendo o período de 2010 a 2020, em língua Portuguesa e Inglesa. Foram excluídos os estudos que não apresentavam claramente o percurso metodológico, relatórios institucionais e os que não apresentavam conteúdo relacionado ao tema e ou contribuíam para o alcance do objetivo desse estudo.

O levantamento bibliográfico foi realizado nas bases de dados: sciELO, Pubmed,PEDro, Lilacs e Google acadêmico, no período de agosto de 2020 a fevereiro de 2021. Foram utilizadas as palavras chaves em português: disfunções sexuais, assoalho pélvico, saúde da mulher. Foram encontrados 78 artigos, e apenas 41 foram selecionados para a pesquisa, após aplicação dos critérios de inclusão e exclusão. Após seleção dos materiais para o referencial, foi realizada leitura e análise minuciosa de cada estudo.

\section{Resultados e Discussão}

\subsection{Gestação}

A gravidez e o parto normal são fatores que predispõe alterações da musculatura do assoalho pélvico, diminuindo a força muscular. O ganho de massa corporal materno e o peso do útero gravídico intensifica a pressão sobre a musculatura do assoalho pélvico (MAP) na gestação. A multiparidade, o parto vaginal, o tempo extenso do segundo período do parto e a episiotomia são causas que reduzem a força dessa musculatura. As modificações fisiológicas sequenciais no decorrer da gestação e o parto lesam o suporte pélvico, o corpo perineal e o esfíncter anal e são causas determinantes, a longo prazo, para o aparecimento das perdas urinárias, disfunções. Dessa forma, para prevenir ou aliviar possíveis problemas se faz necessário o fortalecimento desses músculos, pois contribuirá na manutenção dos órgãos internos em seus lugares, contribuindo também na prevenção de disfunções sexuais e incontinência urinaria e auxiliando no trabalho de parto (Kroetz, 2015).

\subsection{Músculos do Assoalho Pélvico}

Os órgãos externos femininos localizam-se do púbis até o períneo. Conhecido como pudendo, ou vulva, são nomeados os órgãos genitais externos femininos quando considerados coletivamente. São eles: clitóris, monte do púbis, lábios maiores, bulbo do vestíbulo, lábios menores, vestíbulo da vagina e as glândulas vestibulares maiores. Os grandes lábios são duas pregas longitudinais, incluindo a fenda vulvar, já os pequenos lábios ficam na parte interna dos grandes lábios, unidos ao clitóris (Spence, 1991).

O clitóris é um órgão erétil, constituído de um corpo e uma glande, sua estrutura é homologa à porção distal do pênis, sensível ao toque, e fica engurgitado com sangue e rígido quando estimulado, favorecendo para o estímulo e excitação sexual da mulher. A região do púbis, é uma proeminência localizada anteriormente ao púbis, que no decorrer da puberdade se reveste de pelos (Barrote \& Souza, 2000).

Os bulbos do vestíbulo são massas pares de tecido erétil alongados e se localizam-se ao longo dos óstio vaginal sob a cobertura dos músculos bulboesponjosos. O local entre os lábios menores é nomeado de vestíbulo da vagina, entre as paredes da uretra há as aberturas dos ductos das glândulas uretrais (de skene) que secretam muco e nos lados do orifício vaginal estão as glândulas de Bartholin, que fazem a produção do muco para a ver a lubrificação durante o ato sexual (Moore \& Dalley, 2011). 
O assoalho pélvico feminino é dividido em três compartimentos: posterior (reto), anterior (bexiga e uretra), e médio (vagina). Os componentes que auxiliam no suporte do assoalho são: o diafragma pélvico compostos pelos músculos coccígeos e elevadores do ânus, as fáscias pélvicas, e o diafragma urogenital ou espaço perineal profundo onde se localiza o músculo transverso profundo do períneo (Moreno, 2004).

Standring (2010), observou que a musculatura superficial do períneo corresponde as fibras dos músculos transverso superficial do períneo, bulboesponjoso e isquiocavernoso que auxilia nas funções sexuais do assoalho pélvico. O músculo bulboesponjoso tem origem na região anterior do ânus e se anexa no clitóris, realizando a função de ereção do clitóris, contração vaginal e remoção da secreção de glândulas mucosas no decorrer do ato sexual (Fortunato et al., 2014).

Os músculos isquiocavernoso e bulbocavernoso tem como função promover a contração voluntaria e ajudar no orgasmo e na excitação, e são responsáveis pelas contrações rítmicas e involuntárias que acontece no período do orgasmo (Ferreira et al., 2007).

O músculo isquioscavernoso tem como função manter a ereção e tem origem na tuberosidade isquiática que se insere na raiz do clitóris, e os musculos buboesponjoso e bubocavernoso inseridos na base do clitóris auxiliam nessa ereção que são inervados pelo nervo pudendo. (Baracho, 2002).

Figura 1 - Músculos do Assoalho Pélvico.

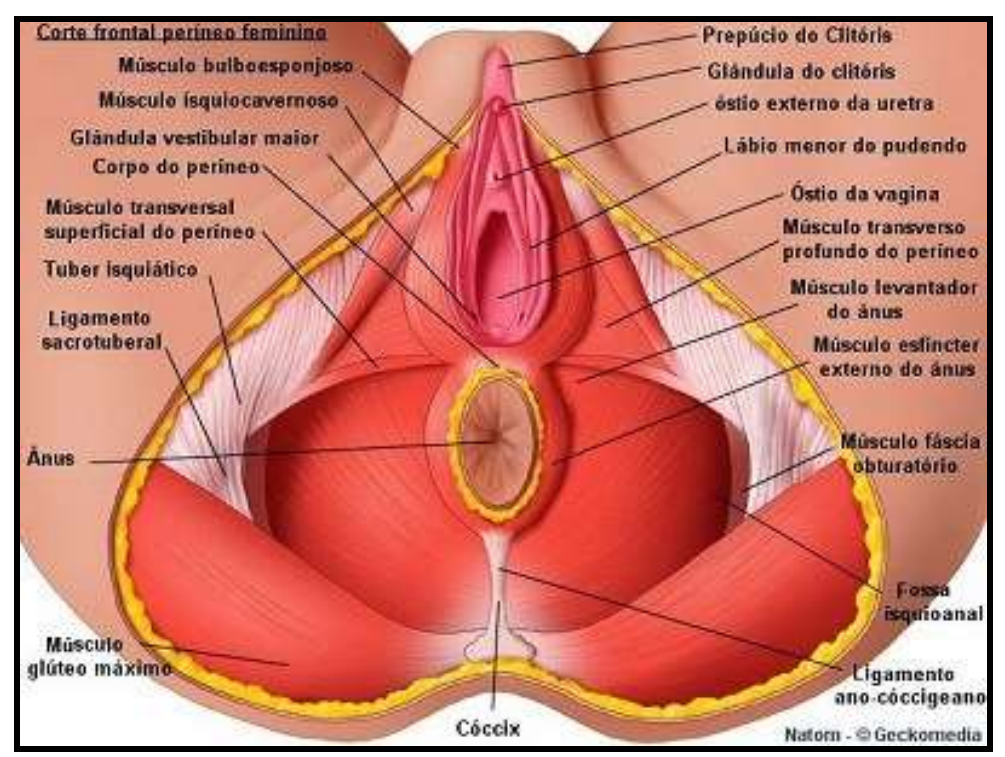

Fonte: http://doulaembelem.blogspot.com/2015/05/o-perineo-e-pelve.html

A musculatura do assoalho pélvico é formada por $70 \%$ de fibras do tipo I (contração lenta), e 30\% de fibras do tipo II (contração rápida) (Silva, 2003; Nolasco, 2007).

\subsection{Exercícios de Fortalecimento para os MAP}

Os exercícios para o fortalecimento da musculatura do assoalho pélvico podem ser realizados antes e depois do parto, pois, devido precisar sustentar o peso gerado pelo bebê e por toda a força exigida no parto essa musculatura tende a se enfraquecer. A realização dos exercícios podem ser feitos através de diversas posições como: decúbito ventral, decúbito dorsal, sentada, posição ortostática, decúbito lateral e de cócoras. A bola suíça é um recurso de grande ajuda para fortalecer os músculos pélvicos, porque ela aumenta o ganho de força da MAP e a percepção sensorial além de, trabalhar outras estruturas musculares (Machado, 2005). 
Os exercícios de fortalecimento da musculatura são aconselhados em toda as fases da mulher, principalmente na fase gestacional para fortalecer o períneo porque durante a gestação a tendência da musculatura é enfraquecer podendo gerar incontinência urinaria por esforço, disfunções sexuais, prolapso genital e o fortalecimento pélvico é determinado por movimentos voluntários e repetidos que fornece aumento da força muscular. Dessa maneira, os exercícios para o fortalecimento dos músculos perineais são benéficos por promover o fortalecimento da musculatura, melhorar a resistência e prevenir disfunções (Baracho, 2014).

Os objetivos diante dos exercícios de fortalecimento para o assoalho pélvico é melhorar a força dos músculos do assoalho pélvico, ganhar mobilidade pélvica e aumentar a sensibilidade clitoriana e perineal e aumentar o fluxo sanguíneo melhorando as fases da resposta sexual, da lubrificação, da excitação, do desejo e do orgasmo, reduzindo as tensões musculares, favorecendo o relaxamento da musculatura, gerando consciência corporal e qualidade sexual (Mendonça; Amaral, 2012), devido a esses fatores fortalecer o assoalho pélvico previne disfunções sexuais, melhora a qualidade e a satisfação sexual, a sensibilidade genital e excitação (Etienne \& Waitman, 2006).

A cinesioterapia é a terapia por meio de movimentos e tem como base movimentos repetidos e voluntários permitindo o aumento da resistência a fadiga, da força muscular, da flexibilidade, da mobilidade, e da coordenação muscular (Amaro; Gameiro, 2001).

Por meio de exercícios de fortalecimento é possível desenvolver o aumento do diâmetro e força de fibras musculares. Isto acontece por a ver o aumento da estimulação da síntese proteica e diminuindo a degradação de proteínas, promovendo o aumento da capacidade de oxidação e de volume mitocondrial (Machado, 2005).

Nolasco et al. (2007), refere que uma ferramenta chave para ser associada no tratamento fisioterápico para o fortalecimento do assoalho pélvico, trabalhando os músculos de forma ativa conforme orientada. A técnicas de cinesioterapia mais utilizada na reeducação perineal, são os exercícios de kegel, originado pelo ginecologista Arnold kegel em 1950, que desenvolveu um programa de exercícios de fortalecimento para o MAP (Moreira; Chaves \& Reis, 2007).

Os princípios básicos dos exercícios para fortalecer a musculatura pélvica é reforçar a resistência uretral e melhorar a sustentação dos órgãos pélvicos (Moura \& Costa, 2006).

A cinesioterapia do assoalho pélvico entende basicamente na execução dos exercícios de Kegel, que determina trabalhar a musculatura perineal para o tratamento da hipertonia ou hipotonia do assoalho pélvico. De maneira geral, consiste em contrair a musculatura do assoalho pélvico com o máximo de força possível, podendo ser contrações fracionadas, sustentadas ou rápidas. Os exercícios de kegel, se consiste nas contrações voluntárias da musculatura do assoalho pélvico, intercalando com as contrações mantidas com o objetivo de estimular as fibras musculares do tipo I e do tipo II do assoalho pélvico. O posicionamento em decúbito dorsal e ventral proporcionam maior facilidade e eficiência na contração do períneo (Latorre, 2010). 
Figura 2 - Exercícios Kegel.

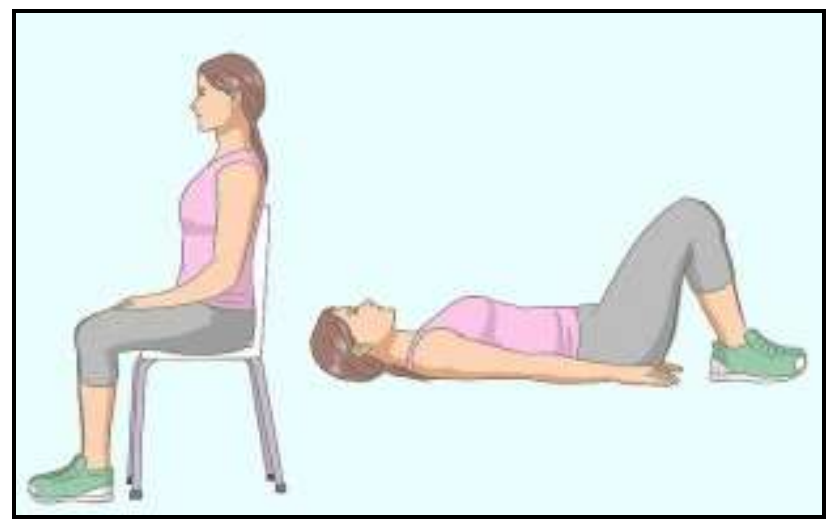

Fonte: https://pt.wikihow.com/Fazer-Exerc\%C3\%ADcios-Kegel

$\mathrm{Na}$ atualidade, a fisioterapia, por meio dos exercícios elaborados por Dr. Kegel por volta de 1949, vem difundido a relevância da ginástica pélvica e, comprovando cientificamente, que os exercícios que trabalham os MAP modificam o trofismo e respostas musculares, potencializam e fortalecem as estruturas internas.

A contração muscular proporciona estímulos proprioceptivos por meio de receptores localizados no ventre e tendões musculares, possibilitando um melhor entendimento da sensação de contração e relaxamento, melhorando suas funções e prevenindo disfunções como por exemplo a incontinência urinaria, a anorgasmia (ausência de orgasmos na relação sexual), prolapso genital e o vaginismo (Buzo et al, 2017).

Os métodos da cinesioterapia do MAP têm como relevância o aumento do músculo levantador do ânus, realizado de forma isolada proporcionando uma melhor conscientização dos músculos agonistas e antagonistas, aumentando a força muscular e da ação reflexa dos músculos do assoalho pélvico durante as atividades de vida diárias. Desse modo, a falta de fortalecimento desse músculo no decorrer da gestação pode provocar lacerações ou estiramentos excessivos no corpo do períneo, região que se encontra entre os esfíncteres vaginal e anal, no decorrer a fase expulsiva, comprometendo a sustentação da porção inferior da parede posterior da vagina (Palma, 2009).

\subsection{Biofeedback}

O biofeedback é um dos aparelhos mais usados na reabilitação da musculatura pélvica, esse aparelho consiste em um retrocontrole biológico, que possibilita a conscientização objetiva de uma função fisiológica inconsciente. Essa conscientização muscular é conseguida através da utilização de um sinal sonoro e/ou visual. Pode-se utilizar o biofeedback através do exame eletromiográfico (EMG); que requer potencial de ação para estimular um movimento, a curva de contração perineal deve ser linear, quantificável e compreensível em ambos os casos (Baracho, 2014).

Aparelho mostra as contrações dos músculos do assoalho pélvico e a sua intensidade é demonstrada por sinais visuais. A sonda inflável é penetrada na vagina, após ser introduzida e insuflada a paciente faz uma contração máxima, onde a intensidade é gravada e visualizada no visor do aparelho em mmHg (Moreno, 2004). 
Figura 3 - Aparelho Biofeedback.

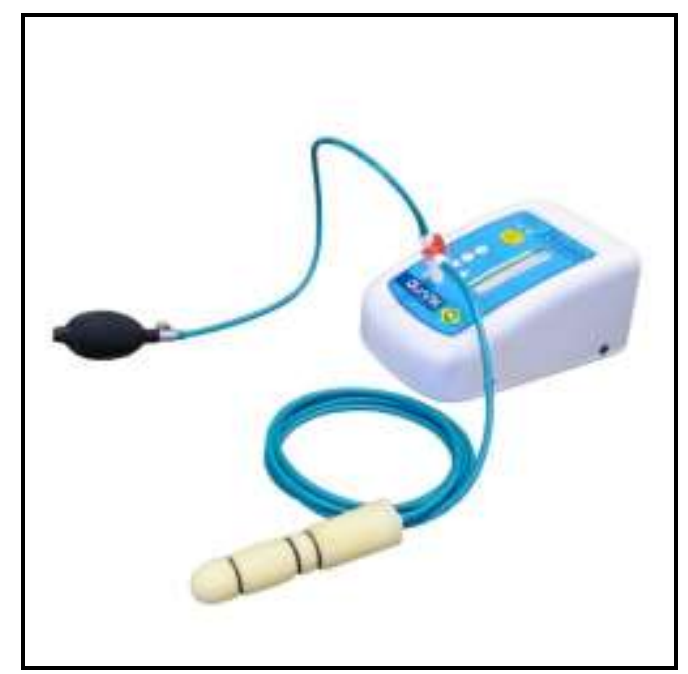

Fonte: https://www.shopfisio.com.br/perina-quark-aparelho-para-urologia-biofeedback-p1057657

O objetivo do biofeedback no tratamento é de auxiliar as pacientes a melhorar ou desenvolver a percepção e controle da musculatura do assoalho pélvico (Moreno, 2004).

\subsection{Eletroterapia}

A utilização da eletroestimulação neuromuscular (EENM) se dá por meio de uma corrente elétrica que estimula a inervação da víscera pélvica ou o suprimento de sua inervação. O intuito que se pretende obter com a EENM é levar a uma resposta terapêutica. A aplicação dessa técnica tem como objetivo de promover a contração passiva da musculatura perineal, mostrando extrema importância na conscientização da contração desta musculatura em pacientes que têm dificuldade de reconhecê-la. A aplicação é feita através de eletrodos endovaginais que estão interligados a um gerador de impulsos elétricos, proporcionando a contração períneal (Silva, 2017).

De acordo com Buzo et al (2017), a eletroestimulação constitui-se na aplicação intravaginal de um dispositivo aproximadamente $7 \mathrm{~cm}$ de comprimento e 2,5 de diâmetro. Esta corrente define como um estímulo elétrico de baixa frequência de 10 a $50 \mathrm{~Hz}$, onde sua intensidade é ajustada a nível da paciente, para que não cause desconforto, sendo eficiente para a percepção da contração da musculatura pélvica.

Figura 4 - Aparelho Eletroestimulação.

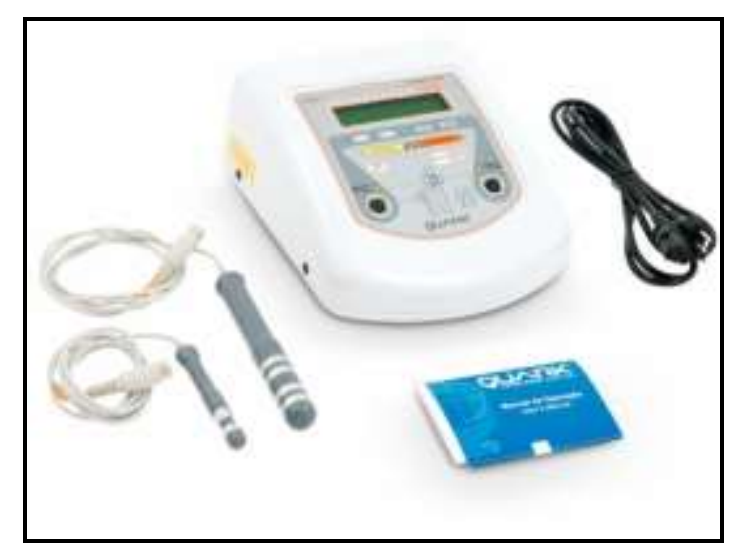

Fonte: https://www.hbfisio.com.br/dualpex-961-uro-eletroestimulacao-ginecologica-pr-108-393794.htm 
A técnica implica a estimulação do nervo pudendo, realizando a contração passiva da musculatura perineal, tornandose muito eficiente na conscientização do assoalho pélvico e reforço muscular.

\subsection{Cones Vaginais}

Os cones vaginais são recursos práticos e simples que ajuda as pacientes a identificar e fortalecer o assoalho pélvico, utilizando dos princípios do biofeedback, pois comprova ser possível aprender a contrair os músculos do assoalho pélvico por meio de sustentar os cones vaginais introduzidos aumentando o peso progressivamente sendo ele um dispositivo de mesmo modelo e volume, com peso que varia de 20 a $120 \mathrm{~g}$ favorecendo o aumento da resistência muscular e da força por meio do recrutamento da musculatura pubiococcígea que auxilia na periferia e na conscientização perineal (Souza et al, 2020).

Figura 4 - Cones Vaginais.

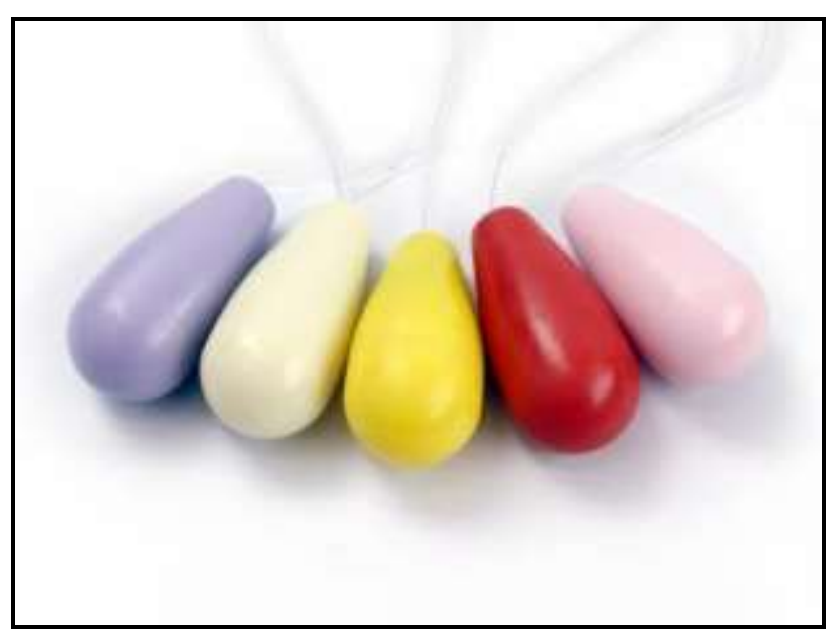

Fonte: http://emilenesilva.com.br/cones-vaginais/

Os cones vaginais proporcionam uma atividade contrátil muscular mais específica e eficiente, mas para que a paciente possa mantê-los na vagina precisa de contração dos MAP's que influenciam na estimulação e recrutamento das fibras do tipo I (contração lenta) e do tipo II (contração rápida), estimulando a propriocepção e estabelecendo o aumento de força muscular do períneo (Silva \& Oliva 2011).

\subsection{Dilatadores Vaginais}

No método de dilatação gradual, são introduzidos dilatadores de silicone ou de material emborrachado lubrificados no canal vaginal como sondas que podem ser insufladas. Inicialmente, os dilatadores precisam ser pequenos; seu tamanho deve ser aumentado gradativamente, à medida que a tolerância da mulher também aumente. O método de dilatação também pode ser realizado usando os dedos (Tomen et al, 2015).

O seguimento de exercícios reside em introduzir e manter sem movimentar o dilatador dentro do canal vaginal no período de 5 a 15 minutos, de acordo com a tolerância na primeira semana, progredindo, na segunda semana, para introdução e retirada parcial e logo após, na terceira semana, para introdução e retirada completa do dilatador no limite de tolerância dolorosa, podendo voltar à etapa anterior se houver muita dificuldade e/ou dor (Araújo \& Scalco, 2019). 
Figura 5 - Dilatadores.

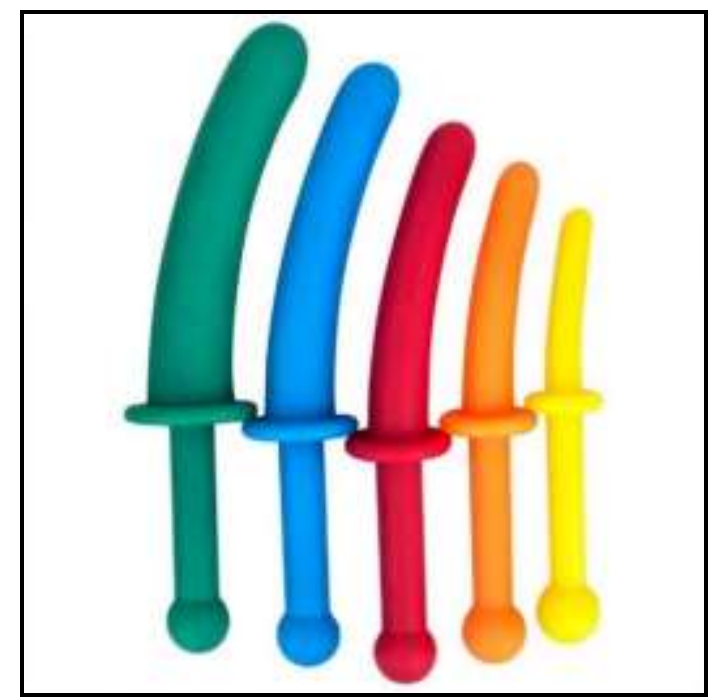

Fonte: https://www.smafisioterapia.com.br/produto/fisioterapia/uroginecologia/dell-dilatadores-vaginais-e-anais-hot-flowers/309/

A utilização dos dilatadores vaginais associados ao método de relaxamento muscular para mulheres em tratamento do vaginismo, tem como objetivo de alongar o introito vaginal e previne a ansiedade pela penetração (Silva \& Abreu, 2014).

\subsection{Terapia Manual}

A massagem perineal constitui-se em uma técnica fisioterapêutica manual de deslizamentos e liberação miofascial de trigger point, na região pélvica. Promove um efeito inibitório da tensão muscular, ocasionando o relaxamento e alongamento progressivo. Melhora o grau de dor, reduz a resistência muscular e facilita a penetração (Lucheti et al, 2019). A técnica deve abranger contato com a pele e as regiões em volta da entrada do canal vaginal, com o intuito de proporcionar um relaxamento progressivo dos músculos pélvicos e dos tecidos adjacentes (Silva e Abreu, 2014).

A massagem é muito eficiente, pois favorece a normalização do tônus muscular através de ações reflexas e mecânicas, e acontece um aumento da circulação sanguínea, da flexibilidade muscular e do fluxo linfático (Delgado et al, 2015).

Figura 6 - Massageador Perineal.

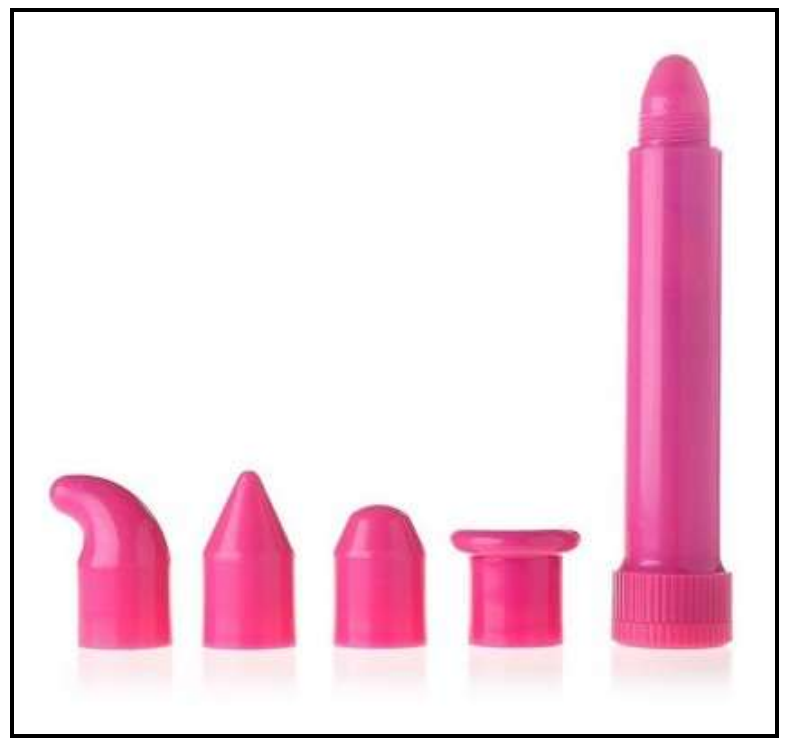

Fonte: https://www.smafisioterapia.com.br/produto/fisioterapia/uroginecologia/peridell-massageador-terapeutico-de-perineo-hot-flowers/2 


\subsection{Pilates}

O método pilates é um seguimento de exercícios que proporciona conscientização corporal, melhora da postura, minimizando quadro álgico na coluna, nas articulares, por meio da liberação de hormônios como: endorfinas e serotoninas. Dentre os benefícios do pilates estão a melhora do alongamento o alinhamento corporal, associando a respiração durante a realização dos exercícios onde o foco é o equilíbrio entre corpo, mente e espírito, enfatizando a postura e a força. Segundo Machado (2005), as gestantes que praticam Pilates tem tendência a ter uma recuperação mais rápida.

Algumas gestantes sofrem com o enfraquecimento da musculatura pélvica é o pilates pode ajudar no fortalecimento dessa musculatura, prevenindo incontinência urinária e fecal, disfunções sexuais. Pelo fato dos exercícios de Pilates recrutar as fibras musculares do assoalho pélvico, o método produz um aumento significativo na força e na contratilidade da musculatura e por esse motivo promove a melhora da função dos MAP, o método pode ser um aliado no tratamento e prevenção de disfunções do assoalho pélvico (Barbosa, 2014).

Figura 7 - Pilates no Assoalho Pélvico.

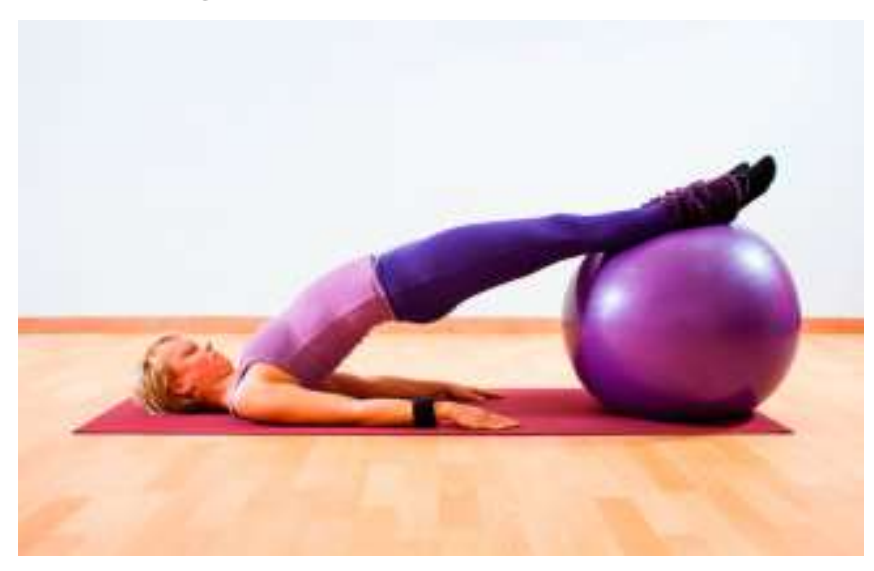

Fonte: https://blogpilates.com.br/vamos-treinar-o-assoalho-pelvico/

\subsection{Efeitos do Fortalecimento da Musculatura do Assoalho Pélvico na Satisfação Sexual}

Os músculos do assoalho pélvico exercem um importante papel nas funções sexuais feminina, entretanto a hipotonicidade e o desuso da musculatura, interfere na função sexual (Piassaroli et al., 2010). Várias situações podem levar ao enfraquecimento dessa musculatura como por exemplo a gestação e o parto (Bianco; Braz, 2010). O propósito da reabilitação fisioterapêutica dos MAP é aumentar a força da musculatura pélvica, aprimorar as atividades fisiológicas da musculatura, consequentemente melhorando a vascularização local estimulando a função sexual (Mendonça; Amaral, 2012).

Tanto a autoconhecimento quanto o fortalecimento do assoalho pélvico são recursos utilizados para tratar disfunções sexuais, aumentando o desejo sexual e a excitação (Etienne; Waitman, 2006). Através dos exercícios de fortalecimento, sobretudo os músculos que compõe o corpo cavernoso do clitóris têm uma melhor resposta sensório-motora na contração involuntária dos músculos do assoalho pélvico durante o orgasmo e é importante enfatizar que os exercícios propiciam melhora na sexualidade, são exercícios terapêuticos e preventivos, para patologias como prolapso genital, disfunções sexuais e incontinência urinária (Passaroli et al., 2010).

\section{Considerações Finais}

A fisioterapia pélvica vem obtendo destaque no tratamento e na prevenção da diminuição da força dos músculos do assoalho pélvico, conseguindo alcançar resultados importantes propiciando benefícios as mulheres. Através de recursos 
fisioterápicos como o fortalecimento para os MAP, incluindo todos os recursos citados acima, proporcionando à mulher uma melhor conscientização corporal, promovendo o autoconhecimento e a reeducação do assoalho pélvico, prevenindo disfunções sexuais e incontinências. Deste modo, pode-se perceber a importância do fortalecimento dessa musculatura, para melhorar satisfação sexual feminina e prevenir disfunções. Portanto, a fisioterapia torna-se cada vez mais participativa e importante no fortalecimento desta musculatura.

O tema abordado possui relevância cientifica por se tratar de um assunto que ainda não é muito abordado na rede cientifica e que tem grande importância para proporcionar a melhora da qualidade de vida das mulheres com qualquer uma disfunção do assoalho pélvico, dessa forma, o estudo demonstra e comprova que precisam ser realizado novas pesquisas para melhorar o embasamento cientifico e estudos que correlacionem a pratica clínica, para potencializar o tratamento fisioterapêutico no fortalecimento dos músculos do assoalho pélvico afim de prevenir disfunções.

\section{Referências}

Amaro, J. L., \& Gameiro, M. O. O (2001). Tratamento Não Cirúrgico Cinesioterapia. In: Rubinstein, I., Clínicas Brasileiras de Urologia Incontinência Urinária na Mulher. 11. Atheneu.

Araujo, T., G. D., \& Scalco, S. C. P. (2019). Transtornos de dor gênito-pélvica/penetração: uma experiência de abordagem interdisciplinar em serviço público. Revista Brasileira de Sexualidade Humana, 30(1), 54-65.

Baracho, E., Baracho, S. M., \& Almeida, L. C (2007). Fisioterapia aplicada a obstetrícia, uroginecologia e aspectos de mastologia. Adaptações do sistema musculoesqueléticos e suas implicações. (4a ed.), Guanabara Koogan. 34 e 35.

Baracho, E., Lotti, R. C. B., \& Reis, A. B (2002). Anatomia Feminina In: Baracho, E. Fisioterapia Aplicada à Obstetrícia, Uroginecologia e Aspectos de Mastologia. (3a ed.), Medsi.

Baracho, E. (2014). Fisioterapia aplicada à saúde da mulher. (5a ed.) Guanabara Koogan,2014. 243-254.

Barbosa, L. J. F (2014). Função dos músculos do assoalho pélvico: comparação entre mulheres praticantes do método pilates e sedentárias. 2014. 67f. Dissertação (Mestrado em Ciência do Movimento Humano) - Escola de Educação Física, Universidade Federal do Rio Grande do Sul - UFRGS, Porto Alegre.

Barrote, M. D., \& Souza, E. L. B. L (2000). Fisioterapia aplicada à obstetrícia e aspectos de neonatologia: Uma visão multidisciplinar. (2a ed.), Health, 25-38.

Bianco, G., \& Braz, M. M. (2010). Efeitos do exercício do assoalho pélvico na sexualidade feminina. Tubarão (SC): UNISUL.

Buzo, D. F. D. C, Cruz, N. C. D., \& Garbin, R. D. F (2017). A importância do fortalecimento da musculatura do assoalho pélvico na satisfação sexual feminina. Faculdades Integradas de Fernandópolis.

Cristina, A. (2020). Vamos Treinar o Assoalho Pélvico. Blog Pilates.

Delgado, A. M., Ferreira, I. S. V., \& Sousa, M. A (2015). Recursos fisioterapêuticos utilizados no tratamento das disfunções sexuais femininas. Revista Cientifica da Escola da Saúde, 4(1), 47-56.

Etienne, M. A., \& Waitman, M. C (2006). Disfunções sexuais femininas: a fisioterapia como recurso terapêutico. LPM, 304p.

Ferreira E. A. (2007). Prevalência das disfunções sexuais femininas em clínica de planejamento familiar de um hospital escola no recife, Pernambuco. Rev. Bras. Saúde Matern. Infant., Recife, 143-150.

Fortunato J. O. (2014). Correlação entre a força dos músculos do assoalho pélvico e a satisfação sexual de mulheres. Cadernos da Escola de Saúde, Curitiba, 2, $143-158$.

HBfisio. Dualpex 961 Uro- Eletroestimulação Genicológica. HB Fisioterapia.

Kroetz, D. C, \& Santos, M. D (2015). Benefícios do método pilates nas alterações musculoesqueléticas decorrentes do período gestacional. Revista Visão Universitária, 3(1), 72-89.

Latorre G. F. S (2010). Exercícios de Kegel.

Lucheti, G. C., Martins, T., \& Fernandes, I (2019). Efeito da massagem perineal no tratamento da disfunção sexual dispareunia. Centro Universitário Uniamérica, Foz do Iguaçu/PR.

Machado (2005). Análise da força muscular em mulheres praticantes de musculação na fase menstrual e pós- menstrual. Revista Digital Vida \& Saúde. Juiz de Fora, 2(1).

Mendonça, C. R. D., Silva, T. M., Arrudai, J. T., Zapata, M. T. A. G., \& Amaral, W. N.D (2012). Função sexual feminina: aspectos normais e patológicos, prevalência no brasil, diagnóstico e tratamento, Femina. 40(4). 
Research, Society and Development, v. 10, n. 2, e56710212894, 2021

(CC BY 4.0) | ISSN 2525-3409 | DOI: http://dx.doi.org/10.33448/rsd-v10i2.12894

Moore, K. L., Dalley, A. F (2011). Anatomia orientada para a clínica. (5a ed.), Guanabara Koogan. 362-365.

Moreira, C. D. V., Chaves, E. R. C., \& Reis, S. M. Y (2007). Estudo comparativo entre a eletroterapia e cinesioterapia no fortalecimento dos músculos do assoalho pélvico entre mulheres nulíparas e multíparas. Belém.

Moreno, A. L (2004). Fisioterapia em uroginecologia. (2a ed.), Manole.

Moura, R. V. A., \& Costa, T. P. B (2006). Avaliação do grau de força do assoalho pélvico em mulheres que apresentam anorgasmia secundária. Universidade da Amazônia- Unama, Belém.

Nolasco et al (2008). Atuação da cinesioterapia no fortalecimento muscular do assoalho pélvico feminino: Revisão bibliográfica.

Nolasco et al (2007). Cinesioterapia no fortalecimento muscular do assoalho pélvico feminino. Fisio \& terapia, (56a ed.).

Palma, P (2009). Aplicações clínicas das técnicas fisioterapêuticas nas disfunções miccionais e do assoalho pélvico. Urofisioterapia, Campinas - SP, s./n., Cap. XXVI, 349-532.

Piassarolli, V. P. et al (2010). Treinamento dos músculos do assoalho pélvico nas disfunções sexuais femininas. Rev Bras Ginecol Obstet., 32(5), 234-40.

Pereira A. S. et al. (2018). Metodologia da pesquisa científica. UFSM.

Polden, M., Mantle, J (2002). Fisioterapia em Ginecologia e Obstetrícia. Santos.

Shopfisio. Perina Quark- Aparelho Para Urologia Biofeedback.

Silva, A. T. C. D (2017). Recursos fisioterapêuticos utilizados no tratamento de incontinência urinária no pós- parto vaginal: revisão narrativa da literatura. Faculdade Escritor Osman da Costa Lins, Vitória de Santo Antão-PE.

Silva, A. M. N., Oliva, L. M. D. P (2011). Exercícios de kegel associados ao uso de cones vaginais no tratamento da incontinência urinária: estudo de caso. Scientia Medica, Porto Alegre, 21(4), 173-176.

Silva, A. P. S., \& Silva, J. S (2003). A importância dos músculos do assoalho pélvico feminino sob uma visão anatômica. Rev. Fisioter Bras. p.11.

Silva, D. J. R. D., \& Abreu, A. H. D O (2014). Recursos fisioterapêuticos para as disfunções sexuais femininas: uma revisão literária. Revista Hórus, v.9, n.1, p.53-66.

Silva, E. (2020). Cones Vaginais. Emilene Silva.

Smafisioterapia. Dell Dilatadores VaginaiS E Anais.

Smafisioterapia. Peridell Massageador Terapêutico de Períneo.

Souza, C., Vaz, M. M. T., Andrade, A., Nunes, E. F. C., \& Latorre, G. F. S (2020). Physical therapy techniques for sexual pain: a systematic review. Revista Médica de Minas Gerais.

Spence, A. P (1991). Anatomia humana básica. (2a ed.), Manole, 620-623.

Tomen, A., Latorre, G. F. S, Fracaro, G., \& Nunes, E. F. C (2015). A fisioterapia pélvica no tratamento de mulheres portadoras de vaginismo. Revista de Ciências Médicas, Campinas, 121- 130.

Trindade, P. O Períneo e a Pelve. Doula em belem.

Wikihow. Preparando-se para fazer os Exercícios Kegel. 